\title{
Comparação da força funcional de membros inferiores e superiores entre idosas fisicamente ativas e sedentárias
}

\author{
Comparison of functional strength of lower and higher limbsamong physically \\ active and sedentary elderly women
}

\section{Resumo}

O objetivo desta pesquisa foi comparar a força funcional dos membros inferiores e superiores entre idosas fisicamente ativas e sedentárias. A amostra foi constituída de 540 voluntárias, divididas em: Grupo Fisicamente Ativo (GFA) e Grupo Sedentário (GS), que se subdividiram em: GFA1 ( $\mathrm{n}=107$ com 61,6 $\pm 1,5$ anos e IMC $=27,1 \pm 3,9)$, GFA2 ( $\mathrm{n}=94$, com $66,7 \pm 1,3$ anos e $\mathrm{IMC}=27,5 \pm 3,6)$, GFA3 ( $\mathrm{n}=82$, com 72,2 $\pm 1,4$ anos $\mathrm{e} \mathrm{IMC}=27,5 \pm 3,8)$, GFA4 ( $\mathrm{n}=75, \mathrm{com} 77,2 \pm 1,3$ anos e IMC $=28,1 \pm 3,9)$, GS1 $(n=36$, com $62,9 \pm 1,2$ anos e $\mathrm{IMC}=27,7 \pm 4,4)$, GS2 ( $\mathrm{n}=39$, com $67,7 \pm 1,3$ anos e IMC $=28,1 \pm 3,1)$, GS3 ( $\mathrm{n}=49$, com $72,5 \pm 1,3$ anos e IMC $=28,3 \pm 2,9)$ e GS4 $(\mathrm{n}=58$, com $77,6 \pm 1,1$ anos e $\mathrm{IMC}=28,3 \pm 3,8)$. Para mensurar a variável em questão, utilizaram-se os testes Chair stand e Arm curl. A estatística foi realizada através dos programas Office Excel 2007 e SPSS16, adotando-se como nível de significância $U<0,05$. Quanto à força funcional de membros inferiores (MMII), todos GFA apresentaram diferenças significativas sobre seus respectivos GS. Para a força funcional de membros superiores (MMSS), apenas os GFA1 e GFA2 apresentaram diferenças significativas sobre os GS1 e GS2. Conclui-se que os programas de atividade física foram capazes de manter, ainda que limitadamente, os níveis de força funcional de MMII e MMSS das idosas fisicamente ativas.

1 Universidade Castelo Branco. Faculdade de Educação e Desporto. Rio de Janeiro, RJ, Brasil.

2 Universidade Federal Fluminense. Centro de Estudos Gerais. Niterói, RJ, Brasil.

3 Universidade Estácio de Sá, Centro de Ciências Biológicas e da Saúde, Farmácia e Fisioterapia. Rio de Janeiro, RJ, Brasil.

4 Escola Nacional de Saúde Pública Sérgio Arouca, Fundação Oswaldo Cruz. Rio de Janeiro, RJ, Brasil.

Correspondência / Correspondence

Fabio Dutra Pereira

E-mail: profabiodutra@hotmail.com
Palavras-chave:

Força Muscular.

Extremidade

Superior. Extremidade Inferior.

Atividade Motora.

Saúde da Mulher.

Mulheres. Idoso.

Força Funcional.

Fisicamente Ativas. Idosas Sedentárias. 


\section{Abstract}

The objective of this research was to compare the functional strength of the higher and lower limbs in physically active and sedentary elderly women. The sample consisted of 540 volunteers, divided into: Physically Active Group (PAG) and Sedentary Group (SG), subdivided into: PAG1 ( $\mathrm{n}=107$ with $61.6 \pm 1.5$ years old and $\mathrm{BMI}=27.1 \pm 3.9)$, PAG2 $(\mathrm{n}=94$, with $66.7 \pm 1.3$ years old and $\mathrm{BMI}=27.5 \pm 3.6)$, PAG3 $(\mathrm{n}=82$, with $72.2 \pm 1.4$ years old and $\mathrm{BMI}=27.5 \pm 3.8)$, PAG4 $(\mathrm{n}=75$, with $77.2 \pm 1.3$ years old and $\mathrm{BMI}=28.1 \pm 3.9)$, SG1 $(n=36$, with $2.9 \pm 1.2$ years old and $B M I=27.7 \pm 4.4)$, SG2 $(n=39$, with $67.7 \pm 1.3$ years old and $\mathrm{BMI}=28.1 \pm 3.1)$, SG3 $(\mathrm{n}=49$, with $72.5 \pm 1.3$ years old and $\mathrm{BMI}=28.3 \pm 2.9)$ and SG4 $(\mathrm{n}=58$, with $77.6 \pm 1.1$ years old and $\mathrm{BMI}=28.3 \pm 3.8)$. To measure the variable in question were used the tests Chairs Stand and Arm Curl. The statistic analysis employed the software

Key words: Muscle Strength. Upper Extremity. Lower Extremity. Motor Activity. Women's Health. Women. Aged. Functional Strength. Physically Active. Elderly Women. Sedentary Elderly Women. OFFICE EXCEL 2007 and SPSS16, adopting as significance level: $U<0.05$. For the functional strength of MMII, all PAG presented significant differences on their respective SG. For the functional strength of MMSS, only PAG1 and PAG2 presented significant differences on the SG1 and SG2. The conclusion is that physical activity programs have been able to maintain, although in limited levels, the functional strength of MMII and MMSS among physically active elderly women.

\section{INTRODUÇÃO}

O envelhecimento é um processo fisiológico de caráter individual, sendo influenciado tanto por fatores genéticos como pelo estilo de vida. Nesse contexto, tal processo afeta diversos fatores, como: força, potência, flexibilidade, equilíbrio, agilidade e resistência aeróbica. ${ }^{1}$ Ao nos reportarmos aos fatores citados inicialmente, podemos atribuí-los à composição da autonomia funcional, que se tornou um dos grandes componentes da saúde do idoso e vem emergindo como forte indicador para a avaliação desta população. ${ }^{2,3}$

Dentre os fatores que compõem a autonomia funcional, destaca-se a força, pois ela está intimamente ligada a realização das atividades da vida diária, manutenção da in- dependência e qualidade de vida dos idosos. ${ }^{4,5}$ Entretanto, com o avançar da idade, essas variáveis se tornam vulneráveis, uma vez que o envelhecimento é um processo que envolve diversas transformações fisiológicas, principalmente no que se diz respeito à perda de autonomia funcional e da integridade do sistema músculo-esquelético. ${ }^{6}$

Uma alternativa para suprimir as consequências do envelhecimento é a adesão a programas de atividade física sistematizada (AFS), pois estes influem positivamente na capacidade funcional, corroborando a hipótese de que a atividade física é um instrumento poderoso para a promoção da saúde do idoso. ${ }^{1}$

Desta forma, ao serem submetidos especificamente a um programa de treinamento 
com sobrecarga, os gerontes garantiriam a manutenção de sua força e equilíbrio, minimizando assim os riscos de quedas e, consequentemente, melhorando a execução das atividades da vida diária (AVDs). ${ }^{5,7} \mathrm{Em}$ contrapartida, quando o envelhecimento se associa ao sedentarismo, acentua-se o declínio das capacidades funcionais dos idosos, diminuindo as funções neuromotoras, podendo acarretar maior incidência de processos patológicos, levando-os a uma condição de fragilidade que aumenta as ocorrências de quedas, chegando a $30 \% \mathrm{com}$ pelo menos uma ocorrência a cada ano e comprometendo sua independência física. ${ }^{8}$

Ao concordar que a força exerce influência fundamental na funcionalidade de tal população, pois é prioritariamente utilizada na realização das AVDs, $, 5,90$ o presente estudo tem por objetivo comparar a força funcional de membros inferiores (MMII) e membros superiores (MMSS) entre idosas fisicamente ativas e sedentárias.

\section{METODOLOGIA}

Sujeitos

O presente estudo teve sua amostra constituída por 540 idosas voluntárias, homogeneizadas por faixa etária ${ }^{11,12} \mathrm{e}$ índice de massa corporal (IMC). ${ }^{13}$ Neste sentido, foram divididas em dois grupos, sendo estes subdivididos respectivamente em: GFA (Grupo Fisicamente Ativo, $n=358$ ) que deu origem para os subgrupos: GFA1 $(n=107$, com idade de $61,6 \pm 1,5$ anos e $\mathrm{IMC}=27,1 \pm 3,9)$, GFA2 ( $\mathrm{n}=94$, com idade de $66,7 \pm 1,3$ anos e $\mathrm{IMC}=27,5 \pm 3,6)$, GFA3 ( $\mathrm{n}=82$, com idade $72,2 \pm 1,4$ anos e $\mathrm{IMC}=27,5 \pm 3,8)$ e GFA4 ( $\mathrm{n}=75$, com idade de $77,2 \pm 1,3$ anos e IMC $=28,1 \pm 3,9)$. E GS (Grupo Sedentário, $n=182$ ), que serviu de base para os subgrupos: GS1 ( $n=36$, com idade de $62,9 \pm 1,2$ anos e $\mathrm{IMC}=27,7 \pm 4,4)$, GS2 $(\mathrm{n}=39$, com idade de $67,7 \pm 1,3$ anos e IMC $=28,1 \pm 3,1)$, GS3 ( $\mathrm{n}=49$, com idade de $72,5 \pm 1,3$ anos e $\mathrm{IMC}=28,3 \pm 2,9)$ e $\mathrm{GS} 4(\mathrm{n}=58$, com idade de $77,6 \pm 1,1$ anos e $\mathrm{IMC}=28,3 \pm 3,8)$.

Como critério de inclusão do GFA, todas as gerontes deveriam ter um nível de participação em seus programas de treinamento físico de pelo menos três sessões semanais num período mínimo de três meses. Cabe ressaltar que as aulas de ginástica localizada eram compostas de dez minutos de aquecimento, 30 minutos destinados ao treinamento cardiorrespiratório e neuromuscular e cinco minutos para o desaquecimento. Para compor o GS, as idosas não poderiam possuir em seu histórico esportivo a participação em qualquer programa sistematizado de treinamento físico por pelo menos seis meses. ${ }^{14}$ Foi considerado como critério de exclusão qualquer tipo de condição patológica aguda ou crônica que pudesse comprometer ou vir a ser fator de impedimento para realização da avaliação da força funcional.

Este estudo teve seu projeto de pesquisa aprovado pelo Comitê de Ética em Pesqui- 
sa Envolvendo Seres Humanos da Universidade Castelo Branco, sob o n ${ }^{\circ}$ 00180/ 2008. Os procedimentos experimentais foram executados dentro das normas éticas previstas na Resolução no 196/96 do Conselho Nacional de Saúde, ${ }^{15}$ e todas participantes assinaram o termo de consentimento livre e esclarecido relativo à avaliação a que seriam submetidas.

\section{Antropometria}

A estatura foi medida com a utilização de um estadiômetro Sanny (Brasil) com acurácia de $0,001 \mathrm{~m}$; a massa corporal foi mensurada com uma balança digital da marca Plena (China), com capacidade de precisão de 0,1Kg; e para realizar a classificação da composição corporal da amostra, foi calculado o IMC adotando-se os seguintes pontos de corte: baixo-peso (IMC < 22), eutrofismo (IMC entre 22 a 27$)$ e sobrepeso (IMC $>27) \cdot{ }^{13}$
Força funcional

$\mathrm{Na}$ aferição desta variável, utilizaram-se os testes Chair stand e Arm curl da bateria de avaliação funcional Sênior Fitness Test, ${ }^{12}$ que assim se descrevem:

Chair stand-Este teste consiste em levantar e sentar na cadeira o maior número de vezes por 30 segundos e tem o objetivo de avaliar o nível de força funcional de membros inferiores (MMII). Em sua execução, a avaliada, partindo da posição sentada na cadeira com os membros superiores fletidos (cotovelo), sobrepostos em "X" e em contato com o tórax, ao sinal verbal do avaliador, iniciará os movimentos de levantar e sentar continuamente até que o sinal de término do teste seja executado. A contagem deve ser feita a partir da fase levantar e não será permitido qualquer forma de auxílio aos MMII na execução dos movimentos. Os valores de referência para esta variável constam da tabela 1 , a seguir. ${ }^{12}$

Tabela 1 - Valores preditos para força funcional de MMII. Rio de Janeiro, RJ. 2008.

\begin{tabular}{lllll}
\hline \multirow{2}{*}{ Chair stand } & G1 & G2 & G3 & G4 \\
& 12 a 17 & 11 a 16 & 10 a 15 & 10 a 15 \\
\hline
\end{tabular}


Arm curl-Este teste baseia-se em realizar flexões de cotovelo o maior número de vezes por 30 segundos e tem o objetivo de avaliar o nível de força funcional de membros superiores (MMSS). Em sua execução, a avaliada, partindo da posição sentada na cadeira com o membro superior de sua prevalência estendido e empunhan- do um implemento de $2,27 \mathrm{Kg}$ (halter adaptado), ao sinal verbal do avaliador, iniciará o movimento de flexão de cotovelo continuamente até que o sinal de término do teste seja executado. A contagem deve ser feita a partir da fase concên trica. Os valores de referência para esta variável são apresentados na tabela $2 .{ }^{12}$

Tabela 2 - Valores preditos para força funcional de MMSS. Rio de Janeiro, RJ. 2008.

\begin{tabular}{lllll}
\hline & G1 & G2 & G3 & G4 \\
Arm Curl & 13 a 19 & 12 a 18 & 12 a 17 & 11 a 17 \\
\hline
\end{tabular}

\section{Tratamento estatístico}

Foram usadas as técnicas de estatística descritiva (média e desvio padrão), no sentido de enquadramento dos grupos estudados nos índices de referência; ${ }^{12}$ para verificar a normalidade da distribuição amostral, utilizou-se o teste Kolmogorov-Smirnov; e na comparação da força funcional de MMII e MMSS intergrupos, efetuouse o teste-U de Mann-whitney, adotandose como nível de significância $U<0,05$. Para realizar o tratamento estatístico deste estudo, foram empregados os programas Office Excel 2007 e SPSS16.

\section{RESULTADOS}

Em primeira análise, nenhum dos grupos estudados possuía distribuição próxima da normalidade, uma vez que, ao realizar o teste Kolmogorov-Smirnov, ambos apresentaram um $\mathrm{p}=0.0001$ tanto para a força funcional de MMII quanto a dos MMSS.

A respeito da força funcional de MMII, deve-se considerar que apenas os GS se encontram abaixo dos índices preditos. ${ }^{12}$

O gráfico 1 demonstra que, ao se comparar as médias intergrupos para esta variável, os GFA apresentaram diferenças significativas de $\mathrm{U}<0,05$ sobre seus respectivos GS. 


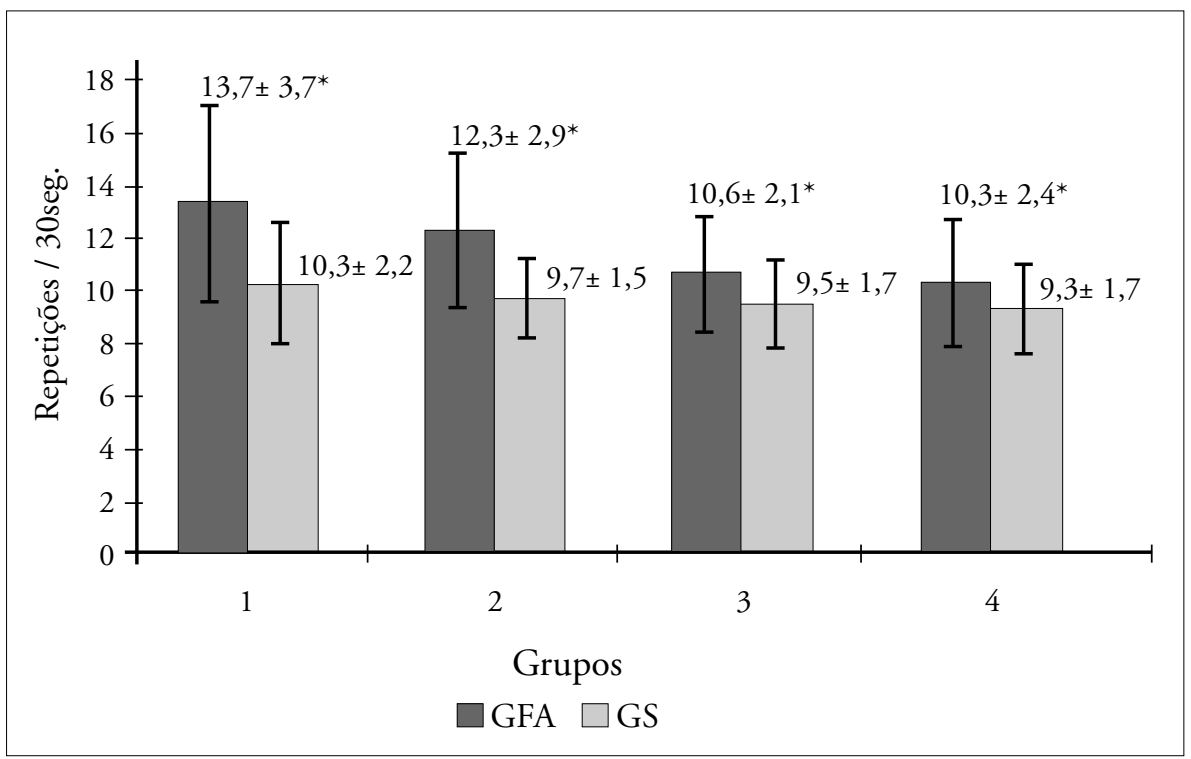

Gráfico 1 - Força funcional de MMII dos GFA e GS.

Quanto à força funcional de MMSS, deve-se considerar que todos GFA e GS encontram-se dentro dos índices preditos. ${ }^{12}$
O gráfico 2 demonstra que, ao se comparar as médias intergrupos para esta variável, somente o GFA1 e 2 apresentaram diferenças significativas de $U<0,05$ sobre os GS1 e 2, respectivamente. 


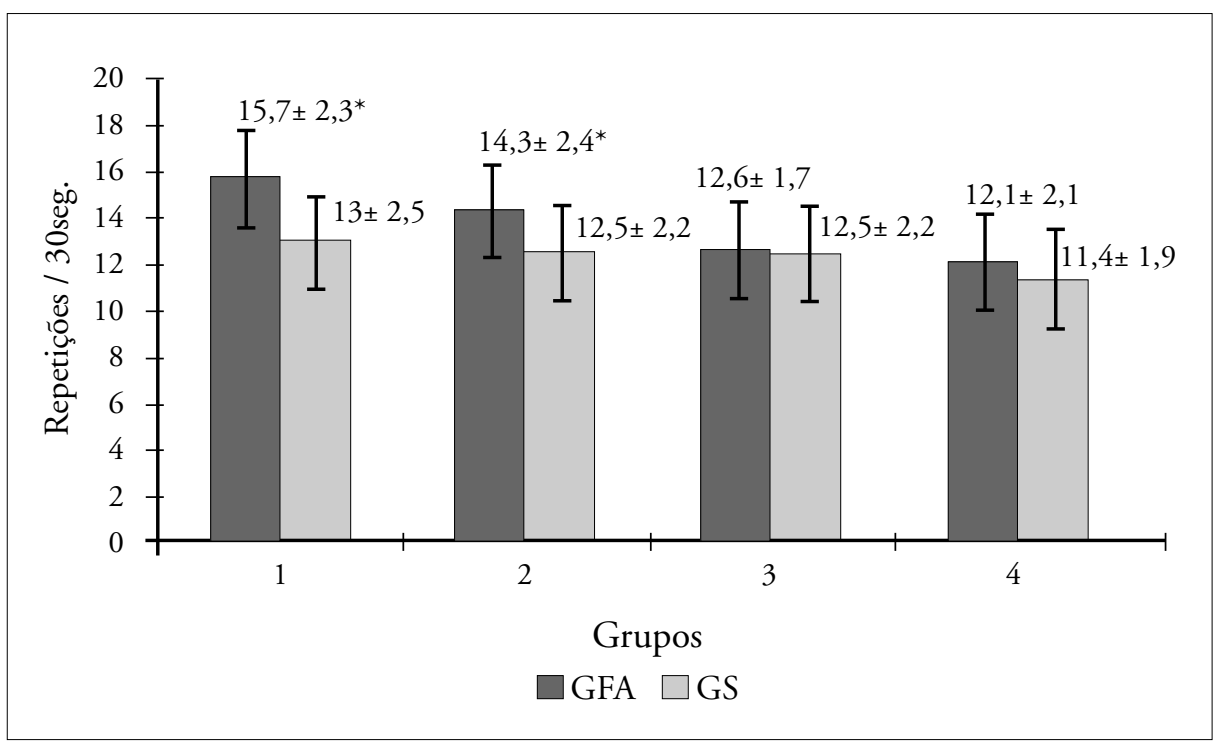

Gráfico 2 - Força funcional de MMSS dos GFA e GS.

\section{DISCUSSÃO}

Tendo como base os resultados expressos no gráfico 1, a força funcional de MMII dos quatro grupos sedentários do presente estudo se encontrou abaixo dos índices de referência. ${ }^{12}$ Tal fato se deu, possivelmente, em função de o sedentarismo ser uma variável importante no processo de diminuição da força $a^{16,17}$ e, quando este é analisado ao atuar sobre a força dos MMII, parece ter efeito mais contundente sobre o sexo feminino,. Outro experimento ${ }^{18}$ demonstrou redução de até $11 \%$ após um período de 12 meses de inatividade física para mulheres ( $\mathrm{n}=11 \mathrm{e} 77,2 \pm 3,4$ anos), modelo que não se dimensiona tanto para os homens $(\mathrm{n}=9 \mathrm{e}$ $77,8 \pm 6,3$ anos), uma vez que eles apresentaram apenas $2 \%$ de diminuição da força dos
MMII pelo mesmo período de inatividade, expressando diferenças significativas entre as amostras pareadas intragêneros na ordem de $\mathrm{p}=0,002$ e $\mathrm{p}=0,052$, respectivamente. Corroborando tal posicionamento, recente pesquisa $^{19}$ feita com 126 gerontes institucionalizados, que foram divididos por sexo e idade - feminino ( $\mathrm{n}=79$ e $84 \pm 6$ anos) e masculino ( $\mathrm{n}=47$ e $81 \pm 8$ anos) - , apresentou em seus resultados $\mathrm{p}<0,05$, quando comparada a força funcional de MMII intergêneros.

Ainda no primeiro gráfico, apesar de os quatro grupos GFA se encontrarem dentro dos valores recomendados, os resultados estão tangenciando os índices mínimos preditos. ${ }^{12}$ Isto representa uma vulnerabilidade dos níveis de força funcional de MMII dos referidos grupos. Essa condição pode ter 
ocorrido pela possível ausência de uma sessão específica de treinamento de força dos programas de atividade física do qual as idosas participavam. Há também, o risco da falha na prescrição da sobrecarga, em que se deve atentar para o número de repetições e de séries, a sequência e os intervalos entre as séries, e os exercícios escolhidos. ${ }^{20,21}$ Quando esses princípios são levados em conta, os efeitos positivos podem ser expressivos, a ponto de uma amostra composta de idosos de ambos gêneros ( $\mathrm{n}=20$ e $76 \pm 8$ anos), ao ser submetida a um programa de atividade física com duração de 11 semanas, conseguir apresentar evolução de $5,7 \pm 6,9$ para $12,7 \pm 6,0$ repetições no teste Chair Stand, demonstrando ganho significativo de força de membros inferiores na ordem de $\mathrm{p}<0,001 .{ }^{22}$ Tendo o mesmo objetivo e também utilizando o referido teste, outro estudo submeteu um grupo de idosas $(\mathrm{n}=18$, com idade entre 75 a 94 anos) a um treinamento de força específico e obteve, em seus resultados, uma diferença de $66 \%$ entre os testes pré e pós-intervenção, correspondendo a $\mathrm{p}<0,05 .{ }^{23}$

Ratificando as informações discutidas até aqui, o gráfico $1 \mathrm{faz}$ menção às diferenças de força funcional de MMII entre os quatro grupos GFA com seus correspondentes GS, evidenciando valores de $\mathrm{U}<0,05 \mathrm{em}$ todas as comparações realizadas no presente estudo. Desta feita, devese considerar que, apesar da fragilidade apresentada pelos GFA quando enquadrados nos índices preditos, ${ }^{12}$ os programas de atividade física que as idosas integravam foram capazes de manter a variável em questão, dentro dos níveis recomendados, podendo amenizar o medo de quedas e minimizar o risco das mesmas. ${ }^{24,25}$

Ao analisar o gráfico 2, os resultados indicam que tanto os quatro GFA como também os GS se encontram dentro dos índices de predição. ${ }^{12} \mathrm{Tal}$ fato ocorreu possivelmente em função de os músculos dos MMSS sofrerem menos com o desuso, ou porque as atividades da vida diária sejam capazes de manter a força funcional dos MMSS dentro dos níveis recomendados para as idosas pertencentes ao GS. Entretanto, cabe ressaltar que a normalidade apresentada deve ser considerada bastante vulnerável quando enquadrada nos limites normativos.

O segundo gráfico demonstra que os grupos GFA1 e 2 apresentaram diferença significativa sobre seus respectivos GS, na ordem de $U<0,05$ em ambas comparações da força funcional de MMSS. Este fato pode ser justificado em função dos efeitos dos programas de atividade física de que as idosas do GFA participavam. Apoiando esta suposição, pesquisa ${ }^{26}$ utilizando o teste de Arm Curl, com o objetivo de avaliar a força funcional dos MMSS de idosos fisicamente ativos de ambos os sexos $(n=169)$, sendo 23 homens e 146 mulheres com idade média de 73 anos, apresentou em seus resultados uma distribuição de frequência de $69,2 \%$ dentro dos limites de predição, $11,8 \%$ acima dos valores normativos e apenas $18,9 \%$ abaixo dos recomendados. Corrobora ou- 
tro estudo, que apresentou em seus resultados uma diferença significativa de $\mathrm{p}<0,05$ ao comparar os efeitos de um programa de atividade física com 12 semanas de duração sobre a força funcional de MMSS de idosas $\left(\mathrm{n}=11\right.$, com idade entre 62 a 80 anos). ${ }^{27}$ Analisando-se ainda o último gráfico, os grupos GFA3 e 4 não apresentaram diferença significativa sobre os grupos GS3 e 4, quando comparados sobre a variável em questão. Tal condição pode ser explicada pela possibilidade de também não ter havido especificidade do treinamento de força nos programas de atividade física que as idosas dos GFA 3 e 4 integravam. ${ }^{20,21}$

Levando-se em conta toda a discussão acerca do gráfico 2 , apesar de as diferenças estatisticamente significativas terem sido somente apresentadas pelos grupos GFA1 e 2 sobre os GS1 e 2, quando comparada a

\section{REFERÊNCIAS}

1. Matsudo SM, et al. Evolução do perfil neuromotor e capacidade funcional de mulheres fisicamente ativas de acordo com a idade cronológica. Revista Brasileira de Medicina do Esporte 2003 nov/dez; 9(6): 377-87.

2. Lima Costa MF, Barreto S, Giatti L. A situação sócioeconômica que afeta igualmente a saúde de idosos e adultos mais jovens no Brasil? Um estudo utilizando dados da Pesquisa Nacional por amostras de domicílios -PNAD/98. Ciência \& Saúde Coletiva 2002; 7(4): 813-24. força funcional dos MMSS intergrupos, ao enquadrar todos os GFA e os GS nos níveis recomendados, ${ }^{12}$ os GS encontraram-se mais próximos dos índices limítrofes inferiores, indicando assim que, de certa forma, os programas de atividade física de que as idosas do GFA participaram foram eficientes na manutenção desta variável.

\section{CONCLUSÃO}

Devido aos resultados obtidos no presente estudo e à compilação realizada em sua discussão, pode-se concluir que, mesmo quando os programas de atividade física não apresentam especificidade do treinamento de força, eles ainda podem proporcionar uma manutenção, ainda que limitada, dos níveis de força funcional de MMII e MMSS nas idosas fisicamente ativas.
3. Amorim FS, Dantas EHM. Autonomia e resistência aeróbica em idosos: efeitos do treinamento da capacidade aeróbica sobre a qualidade de vida e autonomia de idosos. Fitness \& Performance Journal 2002; 1(3): 47-59.

4. Silva NL, Farinatti PTV. Influência de variáveis do treinamento contraresistência sobre a força muscular de idosos: uma revisão sistemática com ênfase nas relações dose-resposta. Revista Brasileira de Medicina do Esporte 2007; 13(1): 60-6. 
5. Batista WO, et al. Análise da autonomia funcional de membros inferiores de idosas iniciantes do programa maturidade ativa / RJ. Revista Augustus 2008 fev; 13(25): 1-6.

6. Marin RS, et al. Acréscimo de $1 \mathrm{~kg}$ aos exercícios praticados por mulheres acima de 50 anos: impacto na aptidão física e capacidade funcional. Revista Brasileira de Ciência e Movimento 2003; 11(1): 53-8.

7. Pedro EM, Bernardes-Amorim D. Análise comparativa da massa e força muscular e do equilíbrio entre indivíduos idosos praticantes e não praticantes de musculação. Conexões 2008; 6(especial): 174-83.

8. Perracini MR, Ramos LR. Fatores associados a quedas em um coorte de idosos residentes na comunidade. Centro de Estudos do Envelhecimento da Universidade Federal de São Paulo. Rev Saúde Pública 2002; 36(6): 709-16.

9. Teixeira DC, et al. Efeitos de um programa de exercício físico para idosas sobre variáveis neuro-motoras, antropométrica e medo de cair. Revista Brasileira Educação Física 2007 abr/jun; 21(2): 107-20.

10. Lemos A, et al. Desempenho da força em idosas após duas intensidades do exercício aeróbio. Revista Brasileira de Medicina do Esporte 2008; 14(1): 28-32.

11. Brasil. Lei n ${ }^{\circ} 10.741$. Dispõe sobre o Estatuto do Idoso e dá outras providências. 2003 out 1. Diário Oficial da União 1(1), [2003 out 3].

12. Rikli RE, Jones CJ. Fitness of older adults. The Journal on Active Aging 2002; 24-30.

13. Cervi A, Franceschini SCC, Priore SE. Análise crítica do uso do índice de massa corporal para idosos. Revista de Nutrição 2005 nov/dez; 18(6): 765-75.
14. Pollock, $M L$, et al. (1998). American College Of Sports Medicine Position Stand: on Fitness. The recommended quantity and quality of exercise for developing and maintaining cardiorespiratory and muscular fitness, and flexibility in healthy adults. Med Sci Sports Exerc 1998 jun; 30(6) 975-91.

15. Brasil. Ministério da Saúde. Conselho Nacional de Saúde. Resolução N.196/96 Regulamento pesquisas envolvendo seres humanos. Diário Oficial da União, 1996 out 10

16. Häkkinen $\mathrm{K}$, et al. Selective muscle hypertrophy, changes in EMG and force, and serum hormones during strength training in older women. J Appl Physiol 2001; 91(2): 569-80.

17. Katula JA, et al. Strength training in older adults: an empowering intervention. Med Sci Sports Exerc 2006; 38(1): 106-11.

18. Capodaglio P, et al. Muscle function and functional ability improves more in community-dwelling older women with a mixed-strength training programme. Age and ageing 2005 mar; 34(2): 141-7.

19. Pedrero-Chamizo R, et al. Gender, age and cognitive score effects on strength in spanish institutionalized elderly. 13th Annual Congress of the European College of Sport Science 2008 jul 9-12; Estoril Portugal. 28p.

20. Wolfe BL, Lemura LM, Cole PJ. Quantitative analysis of single- vs. multiple set programs in resistance training. J Strength Cond Res 2004; (18): 35-47.

21. Manini TM, Druger M, Ploutz-Snyder LL. Misconceptions about strength exercise among older adults. J Aging Phys Act 2005 out; (13): 422-33. 
22. Chaves C, Rodrigues L, Garganta R. Effects of a once-a-week exercise programme in the elderly. 13th annual congress of the European College of Sport Science 2008 jul 9-12; Estoril - Portugal. 28p.

23. Hruda KV, Hicks AL, McCartney N. Training for muscle power in older adults: effects on functional abilities. Can J Appl Physiol 2003; 28(2): 178-89.

24. Freitas MAV, Scheicher ME. Preocupação de idosos em relação a quedas. Revista Brasileira de Geriatria e Gerontologia 2008; 11(1): 57-64.

Recebido: 3/3/2009

Aprovado: 30/7/2009
25. Benedetti TRB, et al. Atividade física e prevalência de quedas em idosos residentes no sul do Brasil. Revista Brasileira de Geriatria e Gerontologia 2008; 11(2): 145-54.

26. Collins K, et al. Funtional fitness, disease and independence in comunity-dwelling older adults in Western Wisconsin. WMJ 2004; 103(1): 42-8.

27. Yamauchi T, et al. Effect of home-based well-rounded exercise in communitydwelling older adults. Journal of Sports Science and Medicine 2005; 4(4): 563-71. 